\title{
Prolonged survival in hepatocarcinoma patients with increased regucalcin gene expression: HepG2 cell proliferation is suppressed by overexpression of regucalcin in vitro
}

\author{
MASAYOSHI YAMAGUCHI ${ }^{1}$, SATORU OSUKA ${ }^{2}$, M. NEALE WEITZMANN ${ }^{3,4}$, \\ BASSEL F. EL-RAYES ${ }^{1}$, MAMORU SHOJI ${ }^{1}$ and TOMIYASU MURATA ${ }^{5}$
}

\author{
Departments of ${ }^{1}$ Hematology and Medical Oncology, and ${ }^{2}$ Neurosurgery, Winship Cancer Institute, \\ Emory University School of Medicine, Atlanta; ${ }^{3}$ The Atlanta Department of Veterans Affairs Medical Center, \\ Decatur; ${ }^{4}$ Division of Endocrinology and Metabolism and Lipids, Department of Medicine, \\ Emory University School of Medicine, 1329 WMRB, Atlanta, GA 30322, USA; \\ ${ }^{5}$ Laboratory of Analytical Neurobiology, Faculty of Pharmacy, \\ Meijo University, Tempaku, Nagoya 468-8503, Japan
}

Received June 3, 2016; Accepted July 29, 2016

DOI: 10.3892/ijo.2016.3669

\begin{abstract}
Hepatocellular carcinoma (HCC) is one of the most common malignant cancers worldwide and ranks third in overall global cancer-related mortality rates. Importantly, in this study gene expression data demonstrate that prolonged survival in HCC patients is associated with increased regucalcin gene expression. Regucalcin has been shown to play a pivotal role as a transcription repressor and diminished expression or activity of regucalcin may play a key role in the development of human carcinogenesis. Indeed, overexpression of regucalcin suppressed the proliferation, cell death, and migration of human HCC HepG2 cells in vitro. Mechanistically, regucalcin induced G1 and G2/M phase cell cycle arrest of HepG2 cells through suppression of multiple signaling pathways including Ras, Akt, MAP kinase and SAPK/JNK and by increasing the tumor suppressors p53 and $R b$. Furthermore, the oncogenes c-fos and c-myc were suppressed by overexpression of regucalcin, and overexpression of regucalcin caused an increase in $\mathrm{p} 21$ and a decrease in NF- $\mathrm{KB}$ p65 and $\beta$-catenin. These findings suggest that regucalcin may play a potential role as a suppressor of human HCC, and that diminished expression of regucalcin may predispose patients to development of HCC.
\end{abstract}

Correspondence to: Dr Masayoshi Yamaguchi, Department of Hematology and Medical Oncology, Winship Cancer Institute, Emory University School of Medicine, C-5054, 1365 C. Clifton Road, NE, Atlanta, GA 30322, USA

E-mail: yamamasa1155@yahoo.co.jp

Key words: regucalcin, hepatocellular carcinoma, cell proliferation, cell death, migration, HepG 2 cells
Overexpression of regucalcin may constitute a novel therapeutic approach to treating HCC.

\section{Introduction}

Hepatocellular carcinoma (HCC), the most common primary liver cancer, is one of the most prevalent malignant diseases worldwide, and the third most common cause of cancer-related death $(1,2)$. Features of HCC are an aggressive cancer with a dismal outcome largely due to metastasis and post-surgical recurrence. HCC originates on a background of cirrhosis, a chronic and diffuse hepatic disease, which results from continuous liver injury and regeneration $(3,4)$. The majority of HCC cases are also related to chronic viral infections including hepatitis $B$ virus or hepatitis $C$ virus, which induces malignant transformation (5-8).

Hepatocarcinogenesis is a multistep process initiated by external stimuli that leads to genetic changes in hepatocytes or stem cells, resulting in proliferation, apoptosis, dysplasia and neoplasia. HCC is a cancer with a poor prognosis. The prognosis of advanced HCC remains poor in spite of the development of novel therapeutic strategies. In recent years, improved knowledge of the oncogenic processes and signaling pathways that regulate tumor cell proliferation, differentiation, angiogenesis, invasion and metastasis has led to the identification of several potential therapeutic targets, which have driven the development of molecularly targeted therapies $(9,10)$. The most effective therapeutic tool for advanced non-resectable HCC, which can slightly improve patient survival, is based on the multikinase inhibitor sorafenib $(11,12)$. New perspectives in cancer treatment have appeared recently with the advent of the microRNAs, a novel class of non-coding small RNAs (13).

Regucalcin, whose gene is localized on the $\mathrm{X}$ chromosome $(14,15)$, plays a potential role as a multi-signaling pathway and transcription repressor in various types of cells and tissues 
$(16,17)$. Regucalcin plays a pivotal role in maintaining intracellular calcium homeostasis, inhibiting various protein kinases and protein phosphatases, suppressing protein synthesis, DNA and RNA synthesis, and regulating gene expression (16-20). Moreover, regucalcin has been found to suppress cell proliferation and apoptotic cell death mediated through various signaling factors $(19,20)$ and has been proposed to play a pivotal role in maintaining cell homeostasis and functioning as a suppressor protein of intracellular signaling systems $(16,17)$.

Noticeably, regucalcin has been shown to be involved in carcinogenesis (21). Regucalcin protein expression was found to be downregulated in the tumor tissues from mammalian and human subjects in vivo (22), suggesting that diminished regucalcin may play a key role in the development of carcinogenesis. Regucalcin gene expression has been demonstrated to be downregulated in rat hepatoma H4-II-E cells in vitro, and enhanced proliferation was reversed by overexpression of regucalcin (23). Moreover, we demonstrated that prolonged overall survival with increased regucalcin expression in pancreatic and breast cancer patients, and that overexpression of regucalcin suppresses the proliferation of human pancreatic cancer MIA PaCa-2 cells and breast cancer MDA-MB-231 cells in vitro $(24,25)$. Regucalcin may play an important role as a suppressor of carcinogenesis.

This study was undertaken to determine the involvement of regucalcin in human $\mathrm{HCC}$, which has not been previously investigated. Importantly, we demonstrated that increased regucalcin gene expression is associated with prolonged survival in HCC patients as evaluated by the analysis of gene expression using the Gene Expression Omnibus (GEO) database (GSE17891) in HCC derived from human patients. Moreover, overexpression of regucalcin was found to suppress the proliferation of human hepatoma HepG2 cells in vitro. Our findings support the view that regucalcin may play a potential role as a suppressor of human $\mathrm{HCC}$, and that diminished expression of regucalcin may predispose patients to development of HCC.

\section{Materials and methods}

Materials. Dulbecco's modified Eagle's medium (DMEM) with $4.5 \mathrm{~g} / 1$ glucose, L-glutamine and sodium pyruvate and antibiotics (penicillin and streptomycin) were purchased from Corning (Mediatech, Inc. Manassas, VA, USA). Fetal bovine serum (FBS) was from Hyclone (Logan, UT, USA). Lipofectamine reagent was obtained from Promega, Madison, WI, USA). Tumor necrosis factor- $\alpha$ (TNF- $\alpha)$ was from R\&D Systems (Minneapolis, MN, USA). Sodium butyrate, roscovitine, sulforaphane, dibucaine, PD98059, lypopolysaccharoide (LPS), Bay K 8644, worthomannin, 5, 6-dichloro-1- $\beta$-Dribofuranosylbenzimidazole (DRB), caspase-3 inhibitor and all other reagents were purchased from Sigma-Aldrich (St. Louis, MO, USA) unless otherwise specified. Gemcytabine was obtained from Hospira, Inc. (Lake Forest, IL, USA). Gemcitabine and caspase-3 inhibitor were diluted in phosphate-buffered saline (PBS) and other reagents were dissolved in $100 \%$ ethanol for use.

Patient datasets. A curated gene expression dataset comprising 35 normal human liver samples and $47 \mathrm{HCC}$ samples were obtained though the Gene Expression Omnibus (GEO) data- base (GSE45436) for analysis of regucalcin expression $(26,27)$. These datasets contained gene expression data derived from the Affymetrix U133_plus2 platform. For microarray analysis, expression and raw expression data (CEL files) were summarized and normalized using the Robust Multi-array Average algorithm and the Bioconductor package affy (http://www. bioconductor.org/packages/2.0/bioc/html/affy.html). The Spotfire Decision Site for Functional Genomics software package (TIBCO Software, Palo Alto, CA, USA) was used for visualization of microarray data. For protein expression analysis, a dataset of immunohistochemistry was obtained from the Human Protein Atlas (HPA) (www.proteinatlas.org), which is a database of proteins in human normal tissues and cancers $(28,29)$. We also evaluated regucalcin expression in 3 tissues of normal liver, especially at the site of hepatocytes, and 6 tissues of HCC in the liver. A dataset of 2 antibodies (HPA029102 and HPA029103) for regucalcin was used in this analysis. Outcome analysis was based on regucalcin mRNA expression in 162 liver tissue samples from HCC patients (GSE10143) (30). A dataset of regucalcin expression and clinical annotation was obtained from SurvExpress (31).

Human hepatoma HepG2 cells. We used HepG2 cells (HB-8065 ${ }^{\mathrm{TM}}$ ) cloned from human HCC, which were obtained from the American Type Culture Collection (ATCC; Rockville, MD, USA). This cell line was isolated from male adolescent (15 years old) with HCC. HepG2 cells were suitable as a transfection host.

Transfection of regucalcin cDNA. HepG2 cells were transfected with pCXN2 vector expressing cDNA encoding human full length (900 bp) regucalcin (regucalcin cDNA/pCXN2) (23). For transient transfection assay, HepG2 cells were grown on 24 -well plates to $70 \%$ confluence. Regucalcin cDNA/pCXN2 and empty pCXN2 vector alone were transfected into HepG2 cells using the synthetic cationic lipid components, a Lipofectamine reagent, according to the manufacturer's instructions (Promega) (23). After resting overnight, Geneticin (500-700 $\mu \mathrm{g} / \mathrm{ml} \mathrm{G418,} \mathrm{Sigma-Aldrich)} \mathrm{was} \mathrm{added} \mathrm{to} \mathrm{culture}$ wells for selection, and cells were cultured for 3 weeks. After that, cells were plated at limiting dilution to isolate transfectants. Multiple surviving clones were isolated, transferred to $35-\mathrm{mm}$ dishes, and grown in the medium without Geneticin. Stable expression of regucalcin was observed in transfectants, and the clone with protein levels of regucalcin increased by 11.2-fold as compared with that of wild-type cells was used in this experiment (Fig. 2A).

Western blotting. HepG2 (wild-type) cells transfected with control vector or regucalcin cDNAs were plated in $35-\mathrm{mm}$ dishes at a density of $1 \times 10^{5}$ cells/well in $2 \mathrm{ml}$ of medium, and cultured in DMEM containing 10\% FBS and 1\% P/S for 3 days. Cells were washed twice with ice-cold PBS and removed from the dish by scraping in cell lysis buffer containing protein inhibitors. Lysed cells were centrifuged and the pellet was homogenized by sonication in $0.1 \mathrm{ml}$ of ice-cold cell lyses buffer containing protein inhibitors. The homogenate was centrifuged for $5 \mathrm{~min}$ at $1,500 \mathrm{~g}$, and the protein concentration of the supernatant was determined for western blotting using bovine serum albumin as a standard. Samples $(30 \mu \mathrm{g})$ 


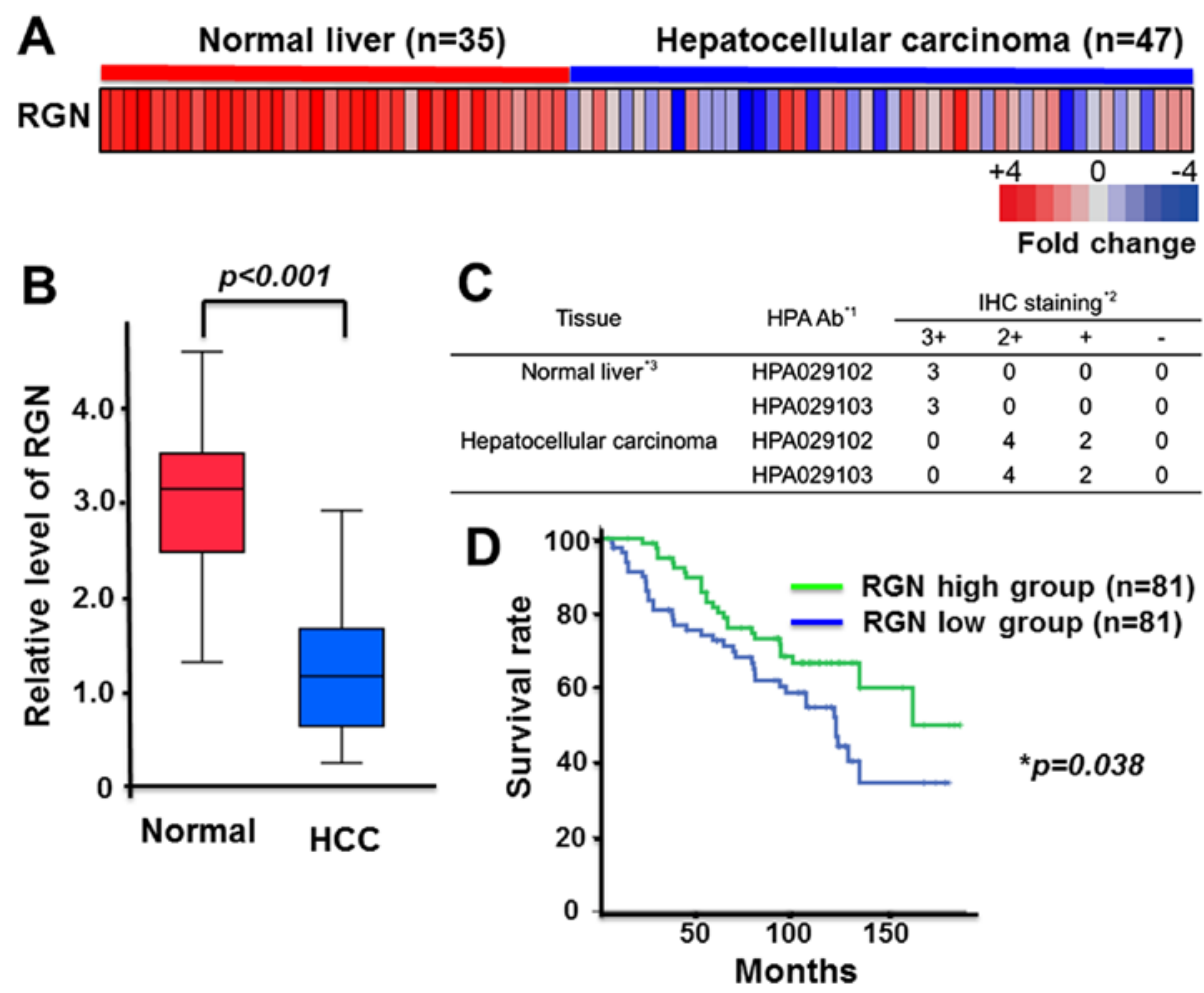

Figure 1. Prolonged survival in hepatocellular carcinoma (HCC) patients with the higher regucalcin gene expression. (A) Microarray expression analysis of regucalcin in 35 normal liver and $47 \mathrm{HCC}(26,27)$. Each colored square on the bottom right represents the relative mean transcript abundance; highest expression is shown in red, average expression in white, and lowest expression is shown in blue. Reduced regucalcin expression weakens survival of HCC patients. (B) Quantification of regucalcin expression in normal liver and HCC similar to that in (A). (C) Degree of immunohistochemical staining for regucalcin into 3 tissues of normal liver and 6 HCC though the immunohistochemistry (IHC) database (The Human Protein Atlas). The results of 2 antibodies are indicated. IHC staining scores: $3+$, storong; $2+$, moderate; +, weak; -, negative. (D) Survival curves for HCC, and survival was longer in high expression as compared with low expression of regucalcin. HCC, hepatocellular carcinoma; HPA, The Human Protein Atlas; IHC, immunohistochemistry; RGN, regucalcin.

of supernatant protein per lane were separated by SDS-PAGE and transferred to nylon membranes for western blotting using antibodies against regucalcin $(22,23)$ and other proteins (Cell Signaling Technology, Danvers, MA, USA). $\beta$-actin was used as a loading control. A minimum of 3 blots from independent experiments were scanned on an Epson Perfection 1660 Photo scanner, and bands quantified using ImageJ. Data from independent experiments were normalized to a percentage of control before averaging.

Cell proliferation assays. Wild-type HepG 2 cells $\left(1 \times 10^{5} / \mathrm{ml}\right.$ per well) and HepG2 cells $\left(1 \times 10^{5} / \mathrm{ml}\right.$ per well) transfected with regucalcin cDNA were cultured using a 24 -well plate in DMEM containing $10 \% \mathrm{FBS}$ and $1 \% \mathrm{P} / \mathrm{S}$ for 1,3 or 6 days in a water-saturated atmosphere containing $5 \% \mathrm{CO}_{2}$ and $95 \%$ air at $37^{\circ} \mathrm{C}(32,33)$. In separate experiments, wild-type HepG2 cells or transfectants were cultured in DMEM containing 10\% FBS and $1 \% \mathrm{P} / \mathrm{S}$ in the presence of either sodium butyrate (10 and $100 \mu \mathrm{M})$, roscovitine (10 and $100 \mathrm{nM})$, sulphoraphane (1 and $10 \mathrm{nM})$, dibucaine $(0.1$ or $1 \mu \mathrm{M})$, Bay $\mathrm{K} 8644(0.1$ or $1 \mu \mathrm{M})$, PD98059 $(1$ or $10 \mu \mathrm{M})$, worthomannin $(0.1$ or $1 \mu \mathrm{M})$, DRB $(0.1$ or $1 \mu \mathrm{M})$, or gemcitabine (50 or $100 \mathrm{nM})$ for 3 days. After culture, cells were detached from each culture dishes and counted.

Cell death assay. Wild-type HepG2 cells ( $1 \times 10^{5} / \mathrm{ml}$ per well) and HepG2 cells $\left(1 \times 10^{5} / \mathrm{ml}\right.$ per well) transfected with regu- calcin cDNA were cultured using a 24-well plate in DMEM containing $10 \% \mathrm{FBS}$ and $1 \% \mathrm{P} / \mathrm{S}$ for 4 days to confluence, and then were cultured for additional 3 days in the presence or absence of either LPS $(0.1$ or $1 \mu \mathrm{g} / \mathrm{ml})$, TNF- $\alpha(0.1$ or $1 \mathrm{ng} /$ $\mathrm{ml})$ or Bay K $8644(0.1$ or $1 \mu \mathrm{M})(34)$. In separate experiments, wild-type HepG2 cells $\left(1 \times 10^{5} / \mathrm{ml}\right.$ per well) or transfectants were cultured for 4 days to confluence, and then were cultured for an additional $24 \mathrm{~h}$ in the presence or absence of either LPS $(1 \mu \mathrm{g} / \mathrm{ml})$ or TNF- $\alpha(1 \mathrm{ng} / \mathrm{ml})$ with or without caspase-3 inhibitor $(10 \mu \mathrm{M})$ for $24 \mathrm{~h}$ (34). After culture, cells were detached from each culture dish for counting.

Cell counting. Cells were recovered from plates by trypsinization of each of culture dishes using $0.2 \%$ trpysin plus $0.02 \%$ EDTA in $\mathrm{Ca}^{2+} / \mathrm{Mg}^{2+}$-free PBS for 2 min at $37^{\circ} \mathrm{C}$, detached cells from dish were collected after centrifugation (32-34). Cells were suspended on PBS solution and stained with eosin. Cells were counted under a microscope using a hemocytometer. For each dish, we took the average of two quantifications.

Migration assay. We used the in vitro scratch assay method for analysis of cell migration in vitro (35). HepG2 cells $\left(1 \times 10^{5}\right.$ cells $\left./ \mathrm{ml}\right)$ of wild-type and transfectants were cultured in DMEM containing 10\% FBS and 1\% P/S for $24 \mathrm{~h}$ using 12-well plates to reach confluence, and then a scratch was created in the cell monolayer. Images were captured at the beginning and at 24 or $48 \mathrm{~h}$ of culture. Cells in the plate were 
A

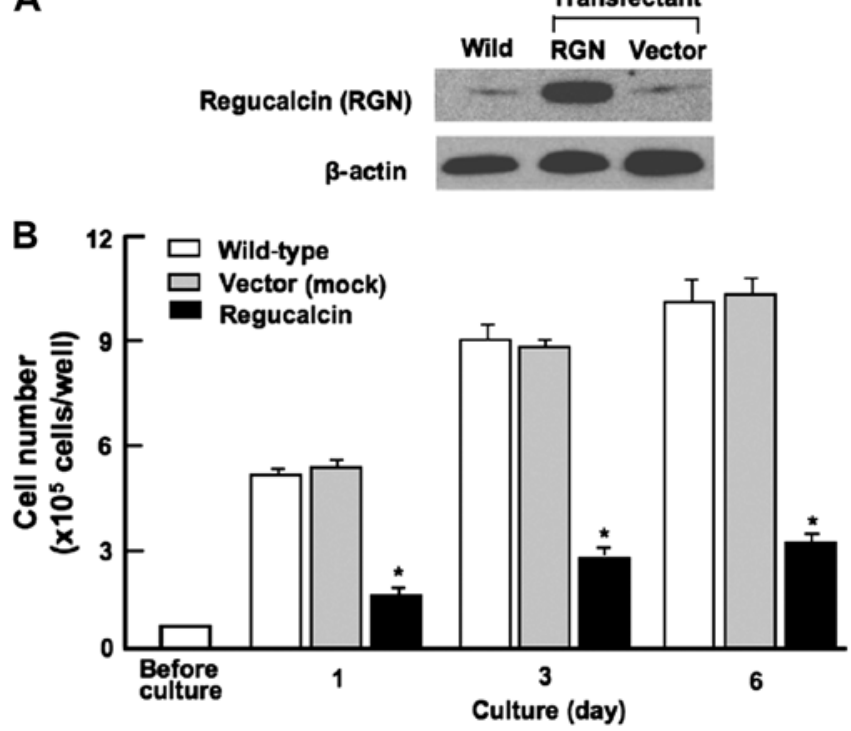

Figure 2. Overexpression of regucalcin suppresses the proliferation in human hepatoma HepG2 cells in vitro. Cells were transfected with regucalcin cDNA vector. (A) Regucalcin content in the cells cultured for 3 days was analyzed by immunoblotting with an anti-regucalcin antibody. Lane 1, wild-type cells (designated as wild). Lane 2, the cells transfected with the regucalcin cDNA/ pCXN2. Lane 3, cells transfected with empty vector/pCXN2 (designated as vector). (B) These cells were cultured in DMEM for 1, 3 or 7 days. After culture, the number of attached cells on a dish was counted. Data are presented as mean \pm SD of 2 replicate wells per data set using different dishes and cell preparation. ${ }^{*} \mathrm{p}<0.001$ versus wild-type (white bar) or control vector (grey bar). One-way ANOVA, Tukey-Kramer post hoc test.

fixed in ice-cold 95\% ethanol and stained with crystal violet (1\% in PBS). After overnight drying, the migration distance was imaged, and cells were counted under a microscope to determine migration.

Statistical analysis. Statistical significance was determined using GraphPad InStat version 3 for Windows XP (GraphPad Software Inc., La Jolla, CA, USA). Multiple comparisons were performed by one-way analysis of variance (ANOVA) with Tukey-Kramer multiple comparisons post hoc test for parametric data as indicated. Survival curves were constructed by Kaplan-Meier analysis and were compared with the log-rank test. Other data were analyzed with the paired or unpaired Student's t-test as performed with IBM SPSS Statistics 18 software (IBM, Chicago, IL, USA http://www.ibm. com). A p-value of $<0.05$ was considered statistically significant.

\section{Results}

Prolonged survival in HCC patients with increased regucalcin gene expression. To understand the involvement of regucalcin in human patients with HCC, we analyzed the expression levels of regucalcin in normal liver tissues and HCC. We analyzed microarray data from the GEO database (GSE45436) (as described in Materials and methods) to evaluate regucalcin expression levels in 35 normal pancreas and 47 HCC patients. Overall regucalcin expression was visually reduced in HCC patients as compared with that of tissues derived from normal liver (Fig. 1A). Quantitative analysis confirmed that expression of regucalcin in HCC patients was remarkably reduced
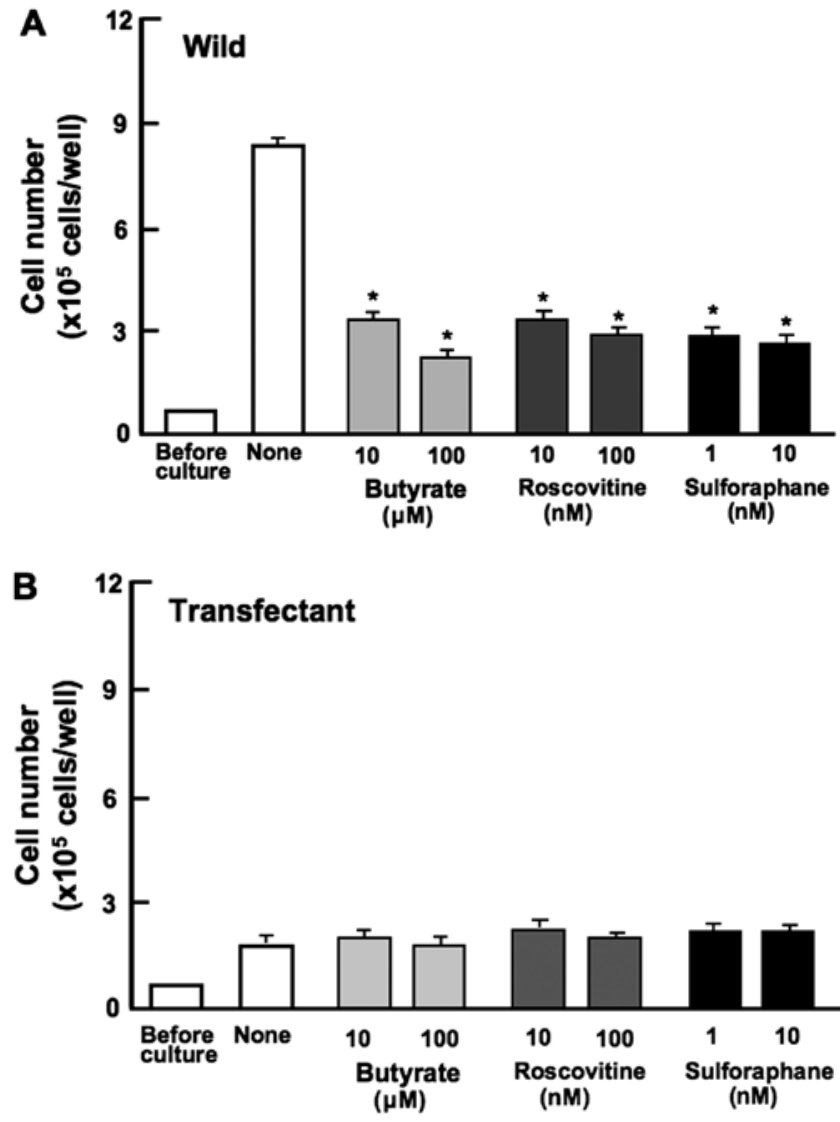

Figure 3. Suppressive effects of regucalcin on the proliferation in HepG2 cells are involved in cell cycle arrest by estimating with cell cycle inhibitors. Wild-type cells (A) or transfectants (B) were culture for 3 days in the absence or presence of butyrate $(10$ and $100 \mu \mathrm{M})$, roscovitine $(10$ and $100 \mathrm{nM})$ or sulforaphane (1 and $10 \mathrm{nM}$ ). After culture, the number of attached cells on dish was counted. Data are presented as mean \pm SD of 2 replicate wells per data set using different dishes and cell preparation. ${ }^{*} \mathrm{p}<0.001$ versus control (none; white bar). One-way ANOVA, Tukey-Kramer post hoc test.

as compared with that in the tissue of normal liver (Fig. 1B). To confirm the reduction of regucalcin protein levels, we checked the expression of regucalcin in 3 normal liver tissue samples and in 6 HCC samples using the immunohistochemistry database (The Human Protein Atlas). Results from 2 independent regucalcin antibodies revealed that the expression of regucalcin in HCC patients was markedly suppressed as compared to that of normal liver (Fig. 1C). Moreover, we compared the clinical outcome between $81 \mathrm{HCC}$ patients with higher regucalcin expression and 81 HCC patients with lower regucalcin expression. The reduction of regucalcin expression was associated with poor prognosis in HCC patients. Higher regucalcin gene expression was found to prolong survival in HCC patients (Fig. 1D). These findings support the notion that suppression of regucalcin gene expression partly contributes to the development of carcinogenesis in human HCC cells and leads to a worse clinical outcome.

Generation of regucalcin-overexpressing Hep G2 cells. HepG2 cells were transiently transfected with empty pCXN2 vector or full length (33-kDa protein) regucalcin/pCXN2 construct using lipofection. Regucalcin protein in transfected cells was found to be increased by 11.2-fold as compared with that of 

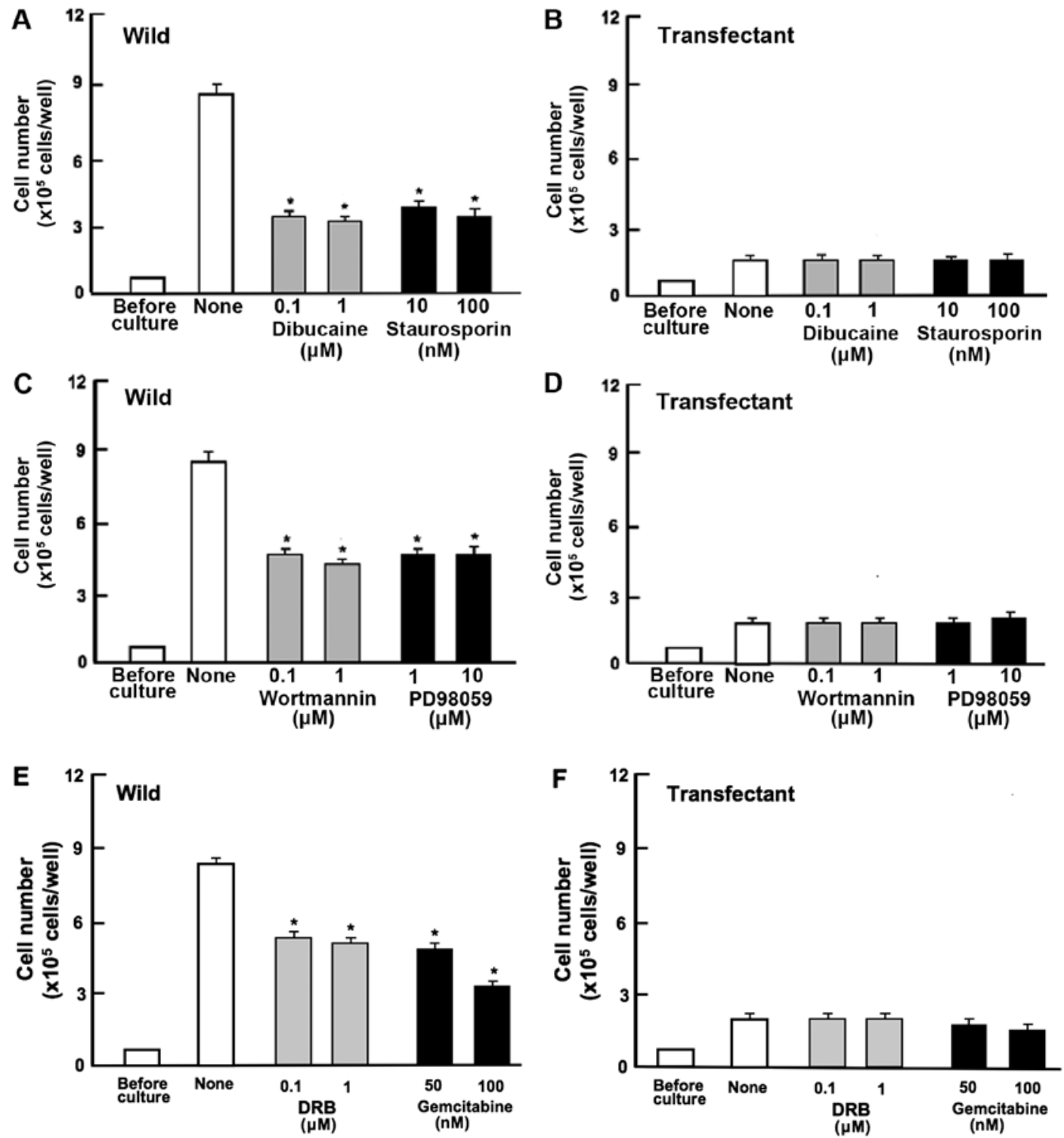

Figure 4. Suppressive effects of regucalcin on the proliferation in HepG2 cells in vitro are exhibited through various signaling pathways. Wild-type cells (A, C and E) or transfectants (B, D and F) overexpressed with regucalcin of full length were cultured in the absence or presence of dibucaine $(0.1$ or $1 \mu \mathrm{M})$, staurosporine $(10$ or $100 \mathrm{nM})$, worthmanin $(0.1$ or $1 \mu \mathrm{M})$, PD98059 $(1$ or $10 \mu \mathrm{M})$, DRB $(0.1$ or $1 \mu \mathrm{M})$ or gemcitabine $(50$ or $100 \mathrm{nM})$ for 3 days. After culture, the number of attached cells on a dish was counted. Data are presented as mean \pm SD of 2 replicate wells per data set using different dishes and cell preparation. " $\mathrm{p}<0.001$ versus control (none; white bar). One-way ANOVA, Tukey-Kramer post hoc test.

the parental wild-type HepG2 cells (Fig. 2A). To determine the effects of the overexpression of endogenous regucalcin on the proliferation of HepG2 cells in vitro, the cancer cells were cultured for 1, 3, and 6 days. Cell proliferation in culture was significantly reduced in regucalcin-overexpressing transfectants at 1,3, and 6 days (Fig. 2B). Thus, overexpression of regucalcin was found to suppress the proliferation of HepG2 cells in vitro.

Suppressive effects of regucalcin overexpression on HepG2 cell proliferation are related to regulation of various signaling pathways. Proliferation in HepG2 cells was determined in the presence of various inhibitors that induce cell cycle arrest in vitro (Fig. 3). Wild-type cells were cultured for 3 days in the presence of butyrate $(10$ and $100 \mu \mathrm{M})$, roscovitine (10 and $100 \mathrm{nM})$ or sulforaphane (1 and $10 \mathrm{nM})(32,36,37)$.
Proliferation of wild-type cells was suppressed in the presence of these inhibitors (Fig. 3A) but not in transfectants (Fig. 3B). This result suggested that endogenous regucalcin induces $\mathrm{G} 1$ and $\mathrm{G} 2 / \mathrm{M}$ phase cell cycle arrest in HepG 2 cells.

Next, to determine the mechanism of regucalcin suppression of cell proliferation, we examined key signaling factors that regulate proliferation. Proliferation in wild-type cells was weakly suppressed by dibucaine $(0.1$ or $1 \mu \mathrm{M})$, an inhibitor of calcium/calmodulin-dependent protein kinases (32), and staurosporine (10 or $100 \mathrm{nM}$ ), a calcium signaling-related inhibitor (32) (Fig. 4A). Blocking these pathways had no effect on the ability of regucalcin to suppress cell proliferation (Fig. 4B). Likewise, worthomannin $(0.1$ or $1 \mu \mathrm{M})$, an inhibitor of phosphatidylinositol 3-kinase (PI3K) (36), and PD98059 (1 or $10 \mu \mathrm{M})$, an inhibitor of extracellular signal-regulated kinase (ERK) and mitogen-activated protein kinase (MAPK) (39) 


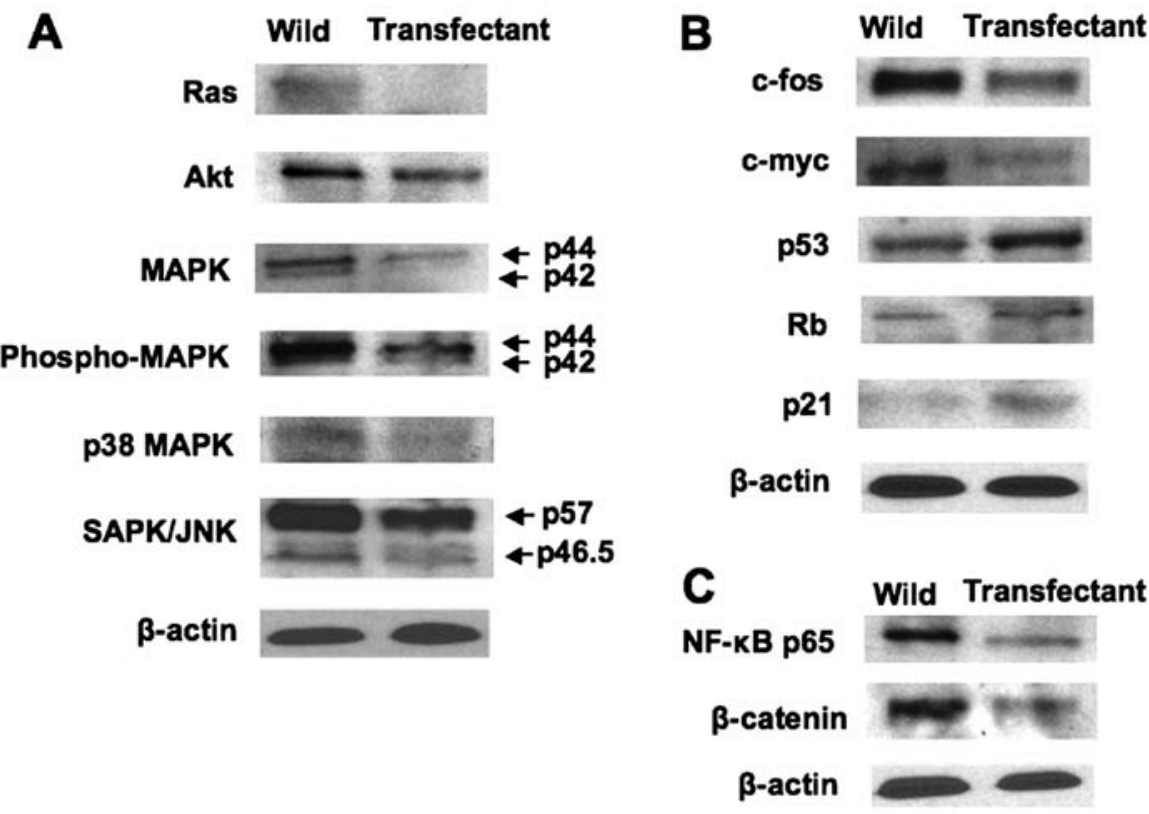

Figure 5. Overexpression of regucalcin regulates various protein levels related to signaling process and transcription activity in HepG2 cells in vitro. Wild-type cells or overexpressing transfectants were cultured in DMEM containing 10\% FBS and 1\% P/S for 3 days. After culture, the cells were removed from the dish with a cell scraper after cell lysis buffer containing protein inhibitors. Samples (30 $\mu \mathrm{g})$ of supernatant protein per lane were separated by SDS-PAGE and transferred to nylon membranes for western blotting using antibodies against various proteins. (A) Cell signaling-related proteins. (B) Tumor-related proteins. (C) Transcription-related proteins. Data are presented as data set using the cell preparation obtained from different dishes with replicate.
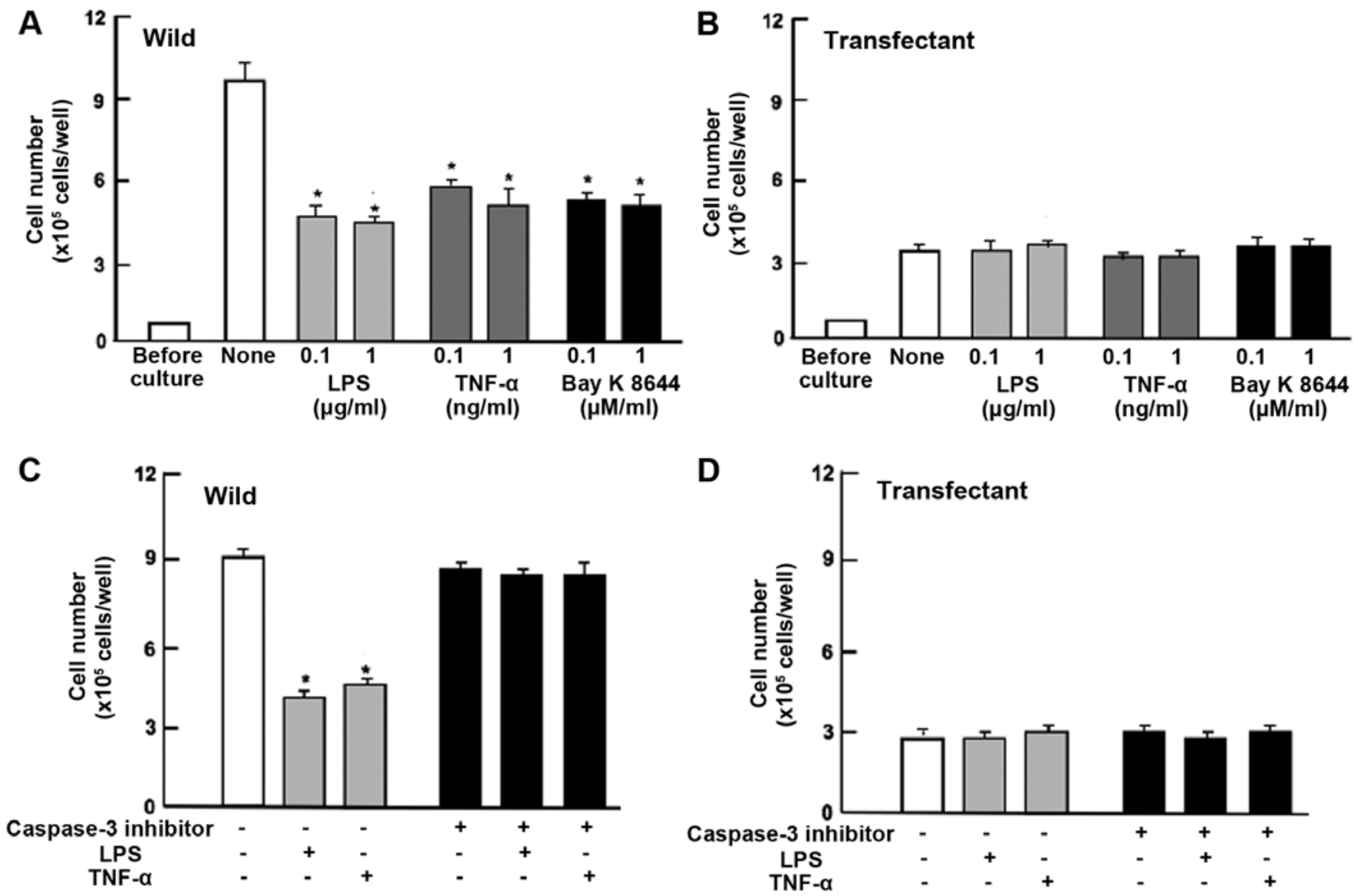

Figure 6. Overexpression of regucalcin prevents cell death induced by stimulation of LPS, TNF- $\alpha$ and Bay K 8644 in HepG2 cells in vitro. Wild-type cells or regucalcin-overexpressing transfectants were cultured for 5 days until confluent, and then the cells were cultured for an additional $24 \mathrm{~h}$ in the presence of LPS $(0.1$ or $1 \mu \mathrm{g} / \mathrm{ml})$, TNF- $\alpha(0.1$ or $1 \mathrm{ng} / \mathrm{ml})$ or Bay K $8644(0.1$ or $1 \mu \mathrm{M})(\mathrm{A}$ or B) or in the presence of LPS $(0.1 \mathrm{or} 1 \mu \mathrm{g} / \mathrm{ml})$ or TNF- $\alpha(0.1 \mathrm{or} 1 \mathrm{ng} / \mathrm{ml})$ with or without caspase-3 inhibitors $(10 \mu \mathrm{M})(\mathrm{C}$ or D). After culture, the number of attached cells on a dish was counted. Data are presented as mean $\pm \mathrm{SD}$ of 2 replicate wells per data set using different dishes and cell preparations. "p $<0.001$ versus control (none; white bar). One-way ANOVA, Tukey-Kramer post hoc test. 


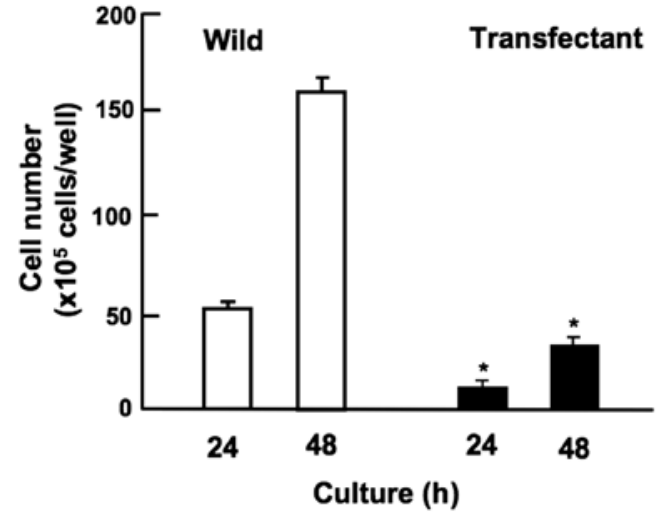

Figure 7. Overexpression of regucalcin suppresses migration of HepG2 cells in vitro. HepG2 cells of wild-type and regucalcin-overexpressing transfectants were cultured for 24 or $48 \mathrm{~h}$ using 12 -well plates until subconfluent, and then a scratch was created in a cell monolayer, capturing the images at 24- or 48-h culture during cell migration. Cells migrated in the space of the scratch was fixed in ethanol and stained with crystal violet. Migrated cells were counted under a microscope. Data are presented as mean \pm SD of 2 replicate wells per data set using different dishes and cell preparations. * $\mathrm{p}<0.001$ versus control (none; white bar). One-way ANOVA, Tukey-Kramer post hoc test.

(Fig. 4C) significantly inhibited cell proliferation in wild-type cells. Suppressive effects of these inhibitors on cell proliferation were not exhibited in transfectants (Fig. 4D). DRB is an inhibitor of transcriptional activity with RNA polymerase II inhibition (40). Gemcitabine is a strong antitumor agent that induces nuclear DNA damage (41). Proliferation of HepG2 cells was suppressed by culture with DRB $(0.1$ or $1 \mu \mathrm{M})$ or gemcitabine (50 or $100 \mathrm{nM}$ ) (Fig. 4E). However, these effects were not seen in transfectants (Fig. 4F).

Overexpression of regucalcin was found to regulate the expression of key proteins related to signaling pathways and transcription activity using western blot analysis. Protein levels of Ras, Akt, MAPK, phospho MAPK, p38-MAPK, and SAPK/JNK were decreased by overexpression of regucalcin (Fig. 5A). These results suggested that overexpression of regucalcin suppresses signaling pathways that are related to activation of ras in HepG2 cells. In addition, overexpression of regucalcin decreased protein levels of c-fos and c-myc in HepG2 cells (Fig. 5B). Protein levels of p53 and $R b$, tumor suppressors, and $\mathrm{p} 21$, cell cycle regulator, were increased by overexpression of regucalcin (Fig. 5B). Moreover, overexpression of regucalcin suppressed protein levels of transcription factor NF-KB p65 and $\beta$-catenin in HepG2 cells (Fig. 5C).

Overexpression of regucalcin prevents cell death induced by various stimulatory factors. To determine the effects of the overexpression of endogenous regucalcin on cell death in HepG2 cells, the cells were cultured for 5 days to confluence and then for an additional $24 \mathrm{~h}$. The number of wild-type cells was decreased in the presence of LPS $(0.1$ or $1 \mu \mathrm{g} / \mathrm{ml})$, TNF- $\alpha$ $(0.1$ or $1 \mathrm{ng} / \mathrm{ml})$ or Bay K $8644(0.1$ and $1 \mu \mathrm{M})$, which is known to induce apoptotic cell death $(20,34)$ (Fig. 6A). Such effects were not seen in the regucalcin-overexpressing HepG2 cells that did not exhibit a significant effect on the death (Fig. 6B). Death of HepG2 cells (wild-type) was also induced once the cells were cultured in the presence of TNF- $\alpha(1 \mathrm{ng} / \mathrm{ml})$ or
Bay K $8644(1 \mu \mathrm{M})$ for 24 and $72 \mathrm{~h}$ (Fig. 6A). Such effects were not seen in the transfectants with regucalcin of full length (Fig. 6B).

To determine whether the preventive effects of regucalcin on cell death are mediated via caspase-3, confluent wild-type cells and transfectants were additionally cultured in the presence of LPS $(1 \mu \mathrm{g} / \mathrm{ml})$ or TNF- $\alpha(1 \mathrm{ng} / \mathrm{ml})$ with or without caspase-3 inhibitors $(10 \mu \mathrm{M})$ for $24 \mathrm{~h}$ (Fig. 6C and D). Effects of LPS or TNF- $\alpha$ on cell death were completely prevented in the presence of caspase-3 inhibitor (Fig. 6C). LPS- or TNF- $\alpha$-induced cell death was not seen in transfectants that were cultured with or without caspase-3 inhibitor (Fig. 6D). These results suggest that endogenous regucalcin prevents cell death due to decreasing the activity of caspase- 3 that activates nuclear DNA fragmentation, inducing apoptotic cell death. Thus, suppressive effects of regucalcin overexpression on the proliferation of HepG2 cells were independent of its effect on cell death.

Overexpression of regucalcin suppresses cell migration. Overexpression of regucalcin suppressed migration of human pancreatic cancer HepG2 cells in vitro (Fig. 7). HepG2 cells of wild-type and transfectants were cultured for $24 \mathrm{~h}$ using 12-well plates to reach subconfluence, and then a scratch was created in a cell monolayer. Cells migrating into the space of the scratch were stained and counted. Overexpression of regucalcin prevented migration of cells into the scratch as compared with that of wild-type cells (Fig. 7).

\section{Discussion}

Using HCC microarray analysis of the GEO database (GSE15471) this study demonstrates overall regucalcin expression is reduced in human HCC patients as compared with that of normal human liver tissues. Furtheremore, patient survival is prolonged with increased regucalcin gene expression while suppressed regucalcin expression was found to be associated with poor prognosis in HCC patients. These findings support the view that suppressed regucalcin gene expression partly contributes to the development or aggressiveness of carcinogenesis in human HCC and leads to a worse clinical outcome.

Overexpression of regucalcin was demonstrated to exhibit anticancer effects in HepG2 cells from human HCC by suppressing the proliferation of cancer cells in vitro. This effect of regucalcin was independent of cell death. In addition, overexpression of regucalcin was found to suppress the migration of HepG2 cells using the scratch assay in vitro, supporting anticancer effects of regucalcin in vitro. Our findings may support the view that regucalcin plays a potential role as a suppressor protein in human HCC cells.

Suppressive effects of regucalcin overexpression on the proliferation of HepG2 cells were not altered in the presence of butyrate, roscovitine or sulphoraphane that induce cellcycle arrest. Roscovitine is a potent and selective inhibitor of the cyclin-dependent kinase cdc2, cdk2m and cdk5 (34). Sulforaphane induces G2/M phase cell cycle arrest (37). Butyrate induces an inhibition of G1 progression (32). Endogenous regucalcin appears to induce G1 and G2/M phase cell cycle arrest in HepG2 cells. Similar data have been 
reported in cloned rat hepatoma H4-II-E cells (32) and normal rat kidney proximal tubular epithelial NRK52E cells (33).

To determine a mechanistic cause of the suppressive effects of regucalcin overexpression on cell proliferation, we used various inhibitors that regulate intracellular signaling pathways. Suppressive effects of regucalcin overexpression on the proliferation in HepG2 cells were not altered in the presence of dibucaine, an inhibitor of calcium/calmodulin-dependent protein kinases (32), worthomannin, an inhibitor of PI3/Akt signaling pathway (38), and PD98059, an inhibitor of ERK/ MAP kinase-related to signaling pathway (39). Regucalcin was shown to exhibit suppressive effects on the proliferation by inhibiting various intracellular signaling pathways, which are related to $\mathrm{Ca}^{2+}$-dependent kinases, PI3/Akt, and ERK/ MAPK in HepG2 cells. Results with western blot analysis confirmed that overexpression of regucalcin led to decrease in protein levels that were involved in the signaling of Ras, Akt and MAPK in HepG2 cells.

In addition, suppressive effects of regucalcin overexpression on cell proliferation were not changed in the presence of DRB, an inhibitor of transcriptional activity with RNA polymerase II inhibition (40). Regucalcin was observed to suppress transcriptional activity in HepG2 cells and to bind to DNA and regulate nuclear gene expression (42). Regucalcin may thus regulate transcriptional activity in HepG2 cells.

Gemcitabine is well known as an antitumor agent that induces nuclear DNA damage, and it is used clinically for the therapy of human pancreatic cancer (41). This agent suppresses cell proliferation and stimulates apoptotic cell death in various types of cancer cells. Suppressive effects of regucalcin overexpression on the proliferation were not potentiated in the presence of gemcitabine in HepG2 cells, suggesting that regucalcin partly acts on pathways related to the action mode of gemcitabine. Regucalcin has been shown to inhibit DNA and RNA synthesis in isolated rat liver nuclei (17-19).

Overexpression of regucalcin was demonstrated to increase the gene expression of p53 and $\mathrm{Rb}$, a suppressor of tumor, and p21, an inhibitor of cell cycle, and suppress the gene expressions of ras, c-jun and c-myc, oncogenes, in cloned rat hepatoma H4II-E cells in vitro (42). Overexpression of regucalcin was also found to increase the protein levels of $\mathrm{p}-53$, $\mathrm{Rb}$ and $\mathrm{p} 21$, and decrease the protein levels of ras, $\mathrm{c}-\mathrm{fos}$ and c-myc in HepG2 cells in vitro. These results may support the view that regucalcin partly mediates a suppressive effect on the proliferation of HepG 2 cells by regulating the expression of tumor suppressor proteins and oncogenes. Interestingly, overexpression of regucalcin was found to decrease $\mathrm{NF}-\kappa \mathrm{B}$ p65 and $\beta$-catenin in HepG2 cells. Regucalcin may suppress signaling process and transcription activity related to $N F-\kappa B$ and $\beta$-catenin in HepG 2 cells. This effect may partly contribute in the revelation of anticancer effects of regucalcin in HCC.

Overexpression of regucalcin was found to prevent cell death induced by various stimulatory factors including LPS, TNF- $\alpha$, and Bay K 8644 in HepG2 cells in vitro, supporting the view that the suppressive effects of regucalcin on the proliferation were not based on cell death. Preventive effect of regucalcin on cell death was not enhanced in the presence of caspase-3 inhibitor. Regucalcin was suggested to prevent cell death through the mechanism by which it increases caspase- 3 activity that activates nuclear DNA fragmentation that induces apoptosis of cells. It is possible that regucalcin directly inhibits caspase-3. In addition, overexpression of regucalcin has been shown to decrease the protein levels of caspase-3 and clevaged caspase-3 in human pancreatic cancer MIA PaCa-2 cells (24) and breast cancer MDA-MB-231 cells (25). Regucalcin has also been shown to directly inhibit calcium-activated endonuclease in isolated rat liver nucleus in vitro (43) and to suppress nitric oxide synthetase activity and to regulate the gene expression of various proteins that are involved in apoptosis in cloned rat hepatoma H4-II-E cells in vitro (34).

In conclusion, this study demonstrates that prolonged survival is associated with increased regucalcin gene expression in HCC patients, and that the overexpression of regucalcin suppresses the proliferation enhanced through various signaling pathways in human hepatoma HepG2 cells in vitro. Our findings may support the view that endogenous regucalcin plays a potential role as a suppressor protein in the development of hepatocarcinogenesis. Overexpression of regucalcin may constitute a novel therapeutic approach to treating HCC.

\section{References}

1. Ferlay J, Shin HR, Bray F, Forman D, Mathers C and Parkin DM: Estimates of worldwide burden of cancer in 2008: GLOBOCAN 2008. Int J Cancer 127: 2893-2917, 2010.

2. El-Serag HB: Hepatocellular carcinoma. N Engl J Med 365: 1118-1127, 2011.

3. Farazi PA and DePinho RA: Hepatocellular carcinoma pathogenesis: From genes to environment. Nat Rev Cancer 6: 674-687, 2006.

4. Dragani TA: Risk of HCC: Genetic heterogeneity and complex genetics. J Hepatol 52: 252-257, 2010.

5. Lee YH and Yun Y: HBx protein of hepatitis B virus activates Jak1-STAT signaling. J Biol Chem 273: 25510-25515, 1998.

6. Andrisani OM and Barnabas S: The transcriptional function of the hepatitis B virus X protein and its role in hepatocarcinogenesis (Review). Int J Oncol 15: 373-379, 1999.

7. Benn J and Schneider RJ: Hepatitis B virus HBx protein activates Ras-GTP complex formation and establishes a Ras, Raf, MAP kinase signaling cascade. Proc Natl Acad Sci USA 91: 10350-10354, 1994.

8. Cha MY, Kim CM, Park YM and Ryu WS: Hepatitis B virus X protein is essential for the activation of Wnt/beta-catenin signaling in hepatoma cells. Hepatology 39: 1683-1693, 2004.

9. Shin JW and Chung Y-H: Molecular targeted therapy for hepatocellular carcinoma: Current and future. World J Gastroenterol 19: 6144-6155, 2013.

10. Singal AG, Marrero JA and Singal AG: Recent advances in the treatment of hepatocellular carcinoma. Curr Opin Gastroenterol 26: 189-195, 2010.

11. Wilhelm SM, Adnane L, Newell P, Villanueva A, Llovet JM and Lynch M: Preclinical overview of sorafenib, a multikinase inhibitor that targets both Raf and VEGF and PDGF receptor tyrosine kinase signaling. Mol Cancer Ther 7: 3129-3140, 2008.

12. Llovet JM, Ricci S, Mazzaferro V, Hilgard P, Gane E, Blanc JF, de Oliveira AC, Santoro A, Raoul JL, Forner A, et al; SHARP Investigators Study Group: Sorafenib in advanced hepatocellular carcinoma. N Engl J Med 359: 378-390, 2008.

13. Callegari E, Elamin BK, Sabbioni S, Gramantieri L and Negrini M: Role of microRNAs in hepatocellular carcinoma: A clinical perspective. Onco Targets Ther 6: 1167-1178, 2013.

14. Shimokawa N and Yamaguchi M: Molecular cloning and sequencing of the cDNA coding for a calcium-binding protein regucalcin from rat liver. FEBS Lett 327: 251-255, 1993.

15. Yamaguchi $M$ : The transcriptional regulation of regucalcin gene expression. Mol Cell Biochem 346: 147-171, 2011.

16. Yamaguchi M: Role of regucalcin in maintaining cell homeostasis and function (Review). Int J Mol Med 15: 371-389, 2005.

17. Yamaguchi M: Regucalcin and cell regulation: Role as a suppressor protein in signal transduction. Mol Cell Biochem 353: 101-137, 2011. 
18. Yamaguchi M: Role of regucalcin in cell nuclear regulation: Involvement as a transcription factor. Cell Tissue Res 354: 331-341, 2013.

19. Yamaguchi M: Suppressive role of regucalcin in liver cell proliferation: Involvement in carcinogenesis. Cell Prolif 46: 243-253, 2013.

20. Yamaguchi M: The anti-apoptotic effect of regucalcin is mediated through multisignaling pathways. Apoptosis 18: 1145-1153, 2013

21. Yamaguchi M: Involvement of regucalcin as a suppressor protein in human carcinogenesis: Insight into the gene therapy. J Cancer Res Clin Oncol 141: 1333-1341, 2015.

22. Murata T and Yamaguchi M: Alternatively spliced variants of the regucalcin gene in various human normal and tumor tissues. Int J Mol Med 34: 1141-1146, 2014.

23. Misawa H, Inagaki S and Yamaguchi M: Suppression of cell proliferation and deoxyribonucleic acid synthesis in the cloned rat hepatoma H4-II-E cells overexpressing regucalcin. J Cell Biochem 84: 143-149, 2001.

24. Yamaguchi M, Osuka S, Weitzmann MN, El-Rayes BF, Shoji M and Murata T: Prolonged survival in pancreatic cancer patients with increased regucalcin gene expression: Overexpression of regucalcin suppresses the proliferation in human pancreatic cancer MIA PaCa-2 cells in vitro. Int J Oncol 48: 1955-1964, 2016.

25. Yamaguchi M, Osuka S, Weitzmann MN, Shoji M and Murata T: Increased regucalcin gene expression extends survival in breast cancer patients: Overexpression of regucalcin suppresses the proliferation and metastatic bone activity in MDA-MB-231 human breast cancer cells in vitro. Int J Oncol 49: 812-822, 2016.

26. Shangguan H, Tan S-Y and Zhang J-R: Bioinformatics analysis of gene expression profiles in hepatocellular carcinoma. Eur Rev Med Pharmacol Sci 19: 2054-2061, 2015.

27. Wang H-W, Hsieh TH, Huang SY, Chau GY, Tung CY, Su CW and Wu JC: Forfeited hepatogenesis program and increased embryonic stem cell traits in young hepatocellular carcinoma (HCC) comparing to elderly HCC. BMC Genomics 14: 736, 2013.

28. Uhlen M, Oksvold P, Fagerberg L, Lundberg E, Jonasson K, Forsberg M, Zwahlen M, Kampf C, Wester K, Hober S, et al: Towards a knowledge-based Human Protein Atlas. Nat Biotechnol 28: 1248-1250, 2010.

29. Uhlén M, Fagerberg L, Hallström BM, Lindskog C, Oksvold P, Mardinoglu A, Sivertsson Å, Kampf C, Sjöstedt E, Asplund A, et al: Proteomics. Tissue-based map of the human proteome. Science 347: 1260419, 2015.

30. Hoshida Y, Villanueva A, Kobayashi M, Peix J, Chiang DY, Camargo A, Gupta S, Moore J, Wrobel MJ, Lerner J, et al: Gene expression in fixed tissues and outcome in hepatocellular carcinoma. N Engl J Med 359: 1995-2004, 2008.

31. Aguirre-Gamboa R, Gomez-Rueda H, Martínez-Ledesma E, Martínez-Torteya A, Chacolla-Huaringa R, Rodriguez-Barrientos A, Tamez-Peña JG and Treviño V: SurvExpress: An online biomarker validation tool and database for cancer gene expression data using survival analysis. PLoS One 8: e74250, 2013.
32. Yamaguchi $M$ and Daimon $Y$ : Overexpression of regucalcin suppresses cell proliferation in cloned rat hepatoma H4-II-E cells: Involvement of intracellular signaling factors and cell cycle-related genes. J Cell Biochem 95: 1169-1177, 2005.

33. Nakagawa T, Sawada N and Yamaguchi M: Overexpression of regucalcin suppresses cell proliferation of cloned normal rat kidney proximal tubular epithelial NRK52E cells. Int J Mol Med 16: 637-643, 2005

34. Izumi T and Yamaguchi M: Overexpression of regucalcin suppresses cell death in cloned rat hepatoma H4-II-E cells induced by tumor necrosis factor-alpha or thapsigargin. J Cell Biochem 92: 296-306, 2004.

35. Liang C-C, Park AY and Guan J-L: In vitro scratch assay: A convenient and inexpensive method for analysis of cell migration in vitro. Nat Protoc 2: 329-333, 2007.

36. Meijer L, Borgne A, Mulner O, Chong JP, Blow JJ, Inagaki N, Inagaki M, Delcros JG and Moulinoux JP: Biochemical and cellular effects of roscovitine, a potent and selective inhibitor of the cyclin-dependent kinases cdc2, cdk2 and cdk5. Eur J Biochem 243: 527-536, 1997.

37. Singh SV, Herman-Antosiewicz A, Singh AV, Lew KL, Srivastava SK, Kamath R, Brown KD, Zhang L and Baskaran R: Sulforaphane-induced G2/M phase cell cycle arrest involves checkpoint kinase 2-mediated phosphorylation of cell division cycle 25C. J Biol Chem 279: 25813-25822, 2004.

38. Serrano-Nascimento C, da Silva Teixeira S, Nicola JP, Nachbar RT, Masini-Repiso AM and Nunes MT: The acute inhibitory effect of iodide excess on sodium/iodide symporter expression and activity involves the PI3K/Akt signaling pathway. Endocrinology 155: 1145-1156, 2014.

39. Chen S, Wang Y, Ruan W, Wang X and Pan C: Reversing multidrug resistance in hepatocellular carcinoma cells by inhibiting extracellular signal-regulated kinase/mitogen-activated protein kinase signaling pathway activity. Oncol Lett 8: 2333-2339, 2014.

40. Palangat M, Grass JA, Langelier MF, Coulombe B and Landick R: The RPB2 flap loop of human RNA polymerase II is dispensable for transcription initiation and elongation. Mol Cell Biol 31: 3312-3325, 2011.

41. Tang SC and Chen YC: Novel therapeutic targets for pancreatic cancer. World J Gastroenterol 20: 10825-10844, 2014.

42. Tsurusaki Y and Yamaguchi M: Role of regucalcin in liver nuclear function: Binding of regucalcin to nuclear protein or DNA and modulation of tumor-related gene expression. Int J Mol Med 14: 277-281, 2004

43. Yamaguchi $\mathrm{M}$ and Sakurai T: Inhibitory effect of calciumbinding protein regucalcin on $\mathrm{Ca}^{2+}$-activated DNA fragmentation in rat liver nuclei. FEBS Lett 279: 281-284, 1991. 